\title{
The comparative analysis of current mirror based CMOS current amplifiers
}

\author{
Mahmut Tokmakcia) \\ Institute of Engineering Science, Erciyes University, \\ Erciyes University, H.B. saglik Hiz. MYO, Kayseri 38039, Turkey \\ a)tokmakci@erciyes.edu.tr
}

\begin{abstract}
In this paper, current mirror based Current Amplifiers (CAs) with differential structure are simulated and compared by PSPICE program using HP $0.8 \mu \mathrm{m}$ CMOS process parameters. The proposed CA designs are based on four well-known current mirror structures such as wilson, improved wilson, cascode, and modified cascode current mirrors. The comparison parameters are power dissipation, bias current, input resistance, output resistance, and cut-off frequency obtained from DC transfer characteristics and AC frequency response of proposed circuits.
\end{abstract}

Keywords: current-mode circuits, current mirrors, current amplifier (CA)

Classification: Integrated circuits

\section{References}

[1] P. E. Allen and D. R. Holberg, CMOS Analog Circuit Design, New York: Oxford University Press, 2002.

[2] G. Palmisano, G. Palumbo, and S. Pennisi, CMOS Current Amplifiers, Boston, MA: Kluwer Academic, 1999.

[3] C. Toumazou, F. J. Lidgey, and D. G. Haigh, Analogue IC Design: The Current-mode Approach, London, Peter Peregrinus, 1990.

[4] P. E. Allen and D. R. Holberg, CMOS Analogue Circuit Design, Oxford, Oxford University Press, 1987.

[5] D. Johns and K. Martin, Analog Integrated Circuit Design, New York: Wiley, 1997.

[6] M. Ismail and T. Fiez, Analog VLSI Signal and Information Processing, New York: McGraw-Hill, 1994.

[7] I. Mucha, "Low-voltage current operational amplifier with a very low current consumption," Proc. IEEE ISCAS'96, vol. 1, pp. 525-528, 1996.

[8] G. Souliotis, A. Chrisanthopoulos, and I. Haritantis, "Current Differential Amplifiers: new circuits and applications," Int. J. Circ. Theor. Appl., vol. 29, pp. 553-574, 2001.

[9] G. W. Roberts and A. S. Sedra, "A General class of current amplifierbased biquadratic filter circuits," IEEE Trans. Circuits Syst. I, Fundam. Theory Appl., vol. 39, no. 4, pp. 257-263, 1992.

[10] Z. Wang, "Analytical determination of output resistance and DC matching errors in MOS current mirrors," IEEE Proc. Circuits Syst., vol. 137, no. 5, pp. 397-404, 1990. 
[11] C. Galanis and I. Haritantis, "An improved current-mode instrumentation amplifier," Proc. IEEE ICECS, vol. 1, pp. 65-68, 1996.

\section{Introduction}

Current Amplifiers (CAs) are attractive blocks for implementing various active filter transfer functions. The achieved maximum frequency of operation of the derived filter topologies is greater than that in case of conventional op-amp RC filters. In addition, operations such as additions/subtraction or scaling are implemented in a very simple way, due to the current-mode nature of the performed signal processing $[1,2,3]$. The Current Amplifiers (CAs) are gaining popularity as alternative building blocks for analog signal processing because of offering the following advantages over the conventional opamps: (a) wide bandwidth which is relatively independent of the closed-loop gain, (b) very high slew-rate, (c) simplicity of realization for various functions with the least possible number of external passive components $[4,5,6]$.

The current mirror is one of the most important building blocks of analog integrated circuits, used as biasing elements and as load devices. Recently, current mirrors (CMs) have been used to replace both op-amps and capacitors implementing Switched-current (SI) filters. Therefore, these designs are required to high-performance current mirrors. For instance, the common mode rejection, supply rejection ratio, and DC gain of an op-amp and other analog circuits such as switched capacitors and converters are strongly dependent on the quality of the current mirrors [3, 4, 5, 6].

In this paper, it is evaluated and compared the simulation characteristics of various (current mirror based) Current Amplifier (CA) designs are based on four well-known current mirror structures such as wilson, improved wilson, cascode, and modified cascode current mirrors. The DC transfer characteristics and AC frequency responses were obtained through computer simulation using the PSPICE program with CMOS $0.8 \mu \mathrm{m}$ HP parameters.

\section{The Description of current mirror based Current Amplifiers}

The topologies of most CAs are based on current mirror structures, interconnected in various ways. The current differential amplifier derives using one or two current mirrors $[1,2,3,4,5]$. An inherent drawback of all of these amplifiers is that, the maximum allowable magnitude of the input current, without distortion at the output, is only a small fraction of the current mirror bias current [6]. Consequently, the dynamic range of the circuits is restricted. This problem can be achieved by using symmetrical current mirrors $[2,5,7]$.

CA structure with differential form consists of three current mirror stages and its symbol as shown in Fig. 1. Stage 2 and 3 are the amplifying stages while Stage 1 is a unity gain current inverter. The CA is symmetrically connected around inputs. Thus, the input signal swing of the amplifier is maximized. All the PMOS and NMOS transistors in the two amplifying 


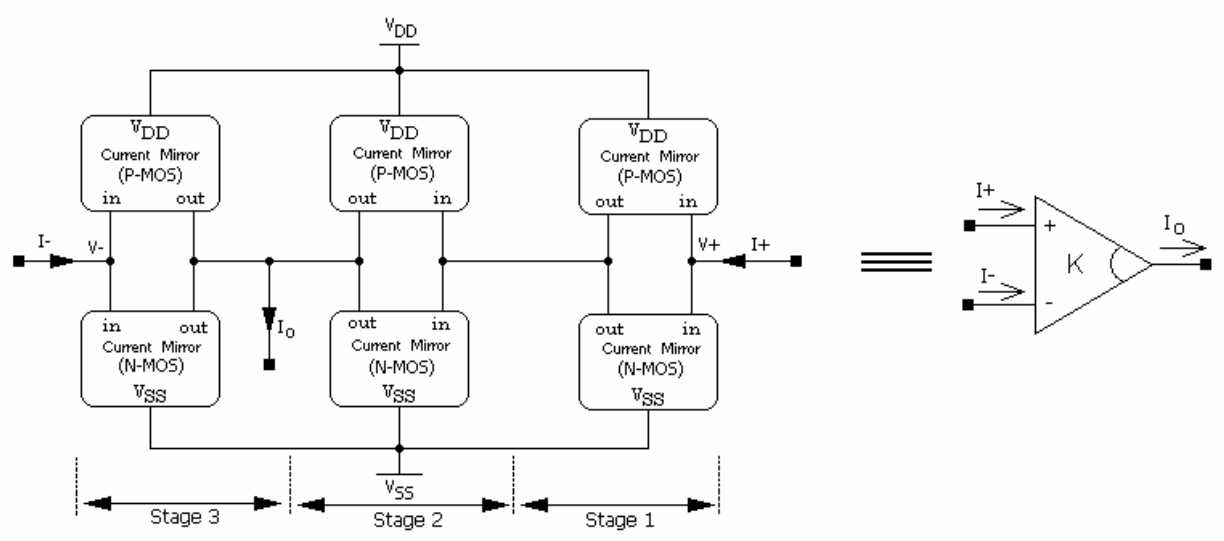

Fig. 1. The block diagram of differential Current Amplifier $(\mathrm{CA})$ structure.

stages are the same dimensions to each other. Therefore, the design of the circuit is very simple. Moreover, since the amplifier topology has modular structure, the CA is easily implemented in IC form without extra processes [2, $8,9]$.

The differential output current of CA circuit is defined as follows:

$$
I_{o}=K\left(I_{+}-I_{-}\right)
$$

Where $\mathrm{K}$ is technological parameter depends on device geometry. Also, $\mathrm{K}$ is obtained by the ratio of the transistor sizes and determined the current gain of the amplifier.

Essentially, the function of a current mirror is to reproduce a current from a given source and the aim of this operation is to have the output of the current mirror identical to the input. In principle, the output current ought to be free from noise and the circuit must have high output impedance. Current mirrors are commonly found in circuits such as amplifiers, comparators, and regulated current sources, etc $[8,9,10,11]$.

In this study, Wilson, improved Wilson, cascode, and modified cascode CMOS current mirror structures are designed with $\mathrm{N}$ and $\mathrm{P}$ type MOS transistors and simulated with HP $0.8 \mu \mathrm{m}$ CMOS process by using PSPICE program. The block diagram of Current Amplifier as shown in Fig. 1 is implemented using each current mirror structure to obtain various comparison parameters.

\section{The Results and Discussions}

Important parameters for the characterization of the Current Amplifiers are: (a) The equivalent input resistances, Ri, and their dependence on the bias current and the input current signal, (b) The current gain, K, and its linearity, (c) The frequency response. Input resistances of proposed Current Amplifiers are defined by

$$
R_{i+}=\frac{V_{+}}{I_{+}} \quad \text { and } \quad R_{i-}=\frac{V_{-}}{I_{-}}
$$


It is $R_{i+}=R_{i-}=R_{i}$ because of input stage of Current Amplifier have symmetrical structure. The equivalent input resistance $R_{i}$ depends on the current mirror bias current $I_{\text {bias }}$. The mirror bias current is related to approximately linear for operation of MOS transistors in saturation region. Also, input resistances of CA can be regarded according to the transconductance Gm of the amplifier in ac small signal operation. It is well known that the transconductance depends on the bias current. However, differential amplifiers do not perform as transconductance amplifiers. It is cannot replace transconductance amplifier since they provide input nodes with relatively low impedances [2]. In our simulation experiments, for specific bias currents $\left(I_{\text {bias }}\right)$, Input current values of all current mirror based CAs used in our study were obtained constant and unaffected by the input signal. However, input resistances of Wilson current mirror based CAs are varied by load resistance values as shown in Table I.

Output resistance is one of the most important performance parameters for a current-mode circuit block. The analytic results of the output resistance are summarized for Wilson, improved Wilson, cascode, and modified cascode current mirrors as follows, respectively. All of equations were obtained by using each small-signal equivalent circuits and identical MOS transistor structures $[1,10]$.

$$
\begin{gathered}
r_{o}=\frac{1}{g_{o}}=\frac{\partial V_{\text {out }}}{\partial I_{\text {out }}} \\
r_{o, \text { Wilson }} \cong g_{m}^{2} r_{d s}^{3} \\
r_{o, \text { imWilson }} \cong\left(1+2 g_{m} r_{d s}+g_{m}^{2} r_{d s}^{2}\right) / g_{m} \\
r_{o, \text { Cascode }} \cong r_{d s}\left(2+g_{m} r_{d s}\right) \\
r_{o, \text { ModCascode }} \cong g_{m}^{2} r_{d s}^{3} /\left(1+g_{m b} r_{d s}\right) \\
g_{m}=\sqrt{\left(2 K^{\prime} W / L\right)\left|I_{D}\right|\left(1+\lambda V_{D S}\right)}
\end{gathered}
$$

Where $g_{m}$ is transconductance parameter of each one of identical MOS transistors, $r_{d s}$ is drain-source resistance (small signal output resistance) of each identical MOS transistor, $r_{d s}=\frac{1}{\lambda I_{D}} . \quad K^{\prime}=\mu C_{o x}, \mu$ is mobility of carriers, $C_{O X}$ is the gate oxide capacitance per unit area, is the channellength modulation factor and $g_{m b}$ is the conductance of the bias current source $I_{B}$. Typical values of nMOS transistor parameters: $K^{\prime}=1.2442 E-4$, $\lambda=0.05 \mathrm{~V}-1, W / L=1.5, V_{D S}=5.0 \mathrm{~V}, I_{D}=100 \mathrm{~A}$, and $g_{m b}=2.5 \mathrm{~A} / \mathrm{V}$.

The current gain $\mathrm{K}$ of the amplifier in Fig. 1 is calculated by the size ratio of current mirror transistors in the two amplifying stages. For unity current gain, both all PMOS transistors and all NMOS transistors are the same dimensions to each other in our simulation experiments. Therefore, the sizes of all PMOS transistors and all NMOS transistors in our simulations have $W=1.6 \mu \mathrm{m}, L=0.8 \mu \mathrm{m}$ and $W=1.2 \mu \mathrm{m}, L=0.8 \mu \mathrm{m}$, respectively. In these simulation experiments, supply voltages are $V_{D D}=-V_{S S}=2.5 \mathrm{~V}$. The cascode current mirror based CA structures have got wide input current range and high output linearity according to Wilson current mirror based CA structures according to simulation results. However, the improved Wilson 


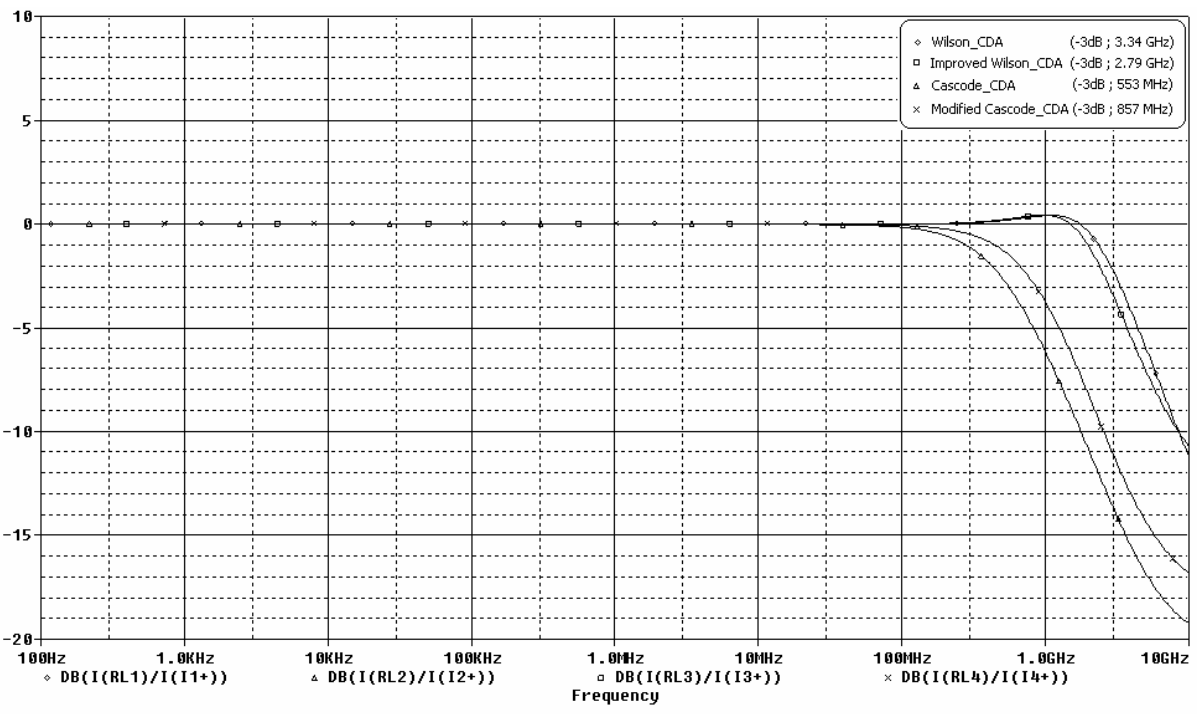

Fig. 2. The overall frequency responses of four CM based CAs.

Table I. Summarized simulation results of current mirror based CA structures.

\begin{tabular}{|c|c|c|c|c|c|c|}
\hline & $\begin{array}{c}\text { Power } \\
\text { Dissipation }\end{array}$ & $\begin{array}{c}\text { Bias } \\
\text { Current }\end{array}$ & Input Resis & ance, $R_{\mathrm{i}}(\mathrm{K} \Omega)$ & $\begin{array}{l}\text { Output } \\
\text { resistance }\end{array}$ & $\begin{array}{c}\text { Cut off } \\
\text { Frequency }\end{array}$ \\
\hline & $\mathrm{PD}(\mathrm{mWV})$ & $\mathrm{I}_{\mathrm{bias}}(\mu \mathrm{A})$ & $\mathrm{R}_{\mathrm{L}}=\mathrm{l} \mathrm{K} \Omega$ & $\mathrm{R}_{\mathrm{L}}=10 \mathrm{~K} \Omega$ & $\mathrm{R}_{0}(\mathrm{~K} \Omega)$ & $f_{c}($ for $\mathrm{K}=\mathrm{l})$ \\
\hline Wilson CM based CA & 4.70 & 70.829 & 12.915 & 15.320 & 388 & $3.34 \mathrm{GHz}$ \\
\hline Cascode CM based CA & 3.31 & 43.411 & 15.962 & 15.962 & 763 & $553 \mathrm{MHz}$ \\
\hline $\begin{array}{lll}\text { Improved Wilson } & \text { CM } \\
\text { based CA } & & \end{array}$ & 2.76 & 59.166 & 21.276 & 25.111 & 729 & $2.79 \mathrm{GHz}$ \\
\hline $\begin{array}{l}\text { Modified Cascode CM } \\
\text { based CA }\end{array}$ & 6.33 & 37.665 & 14.523 & 14.523 & 214 & $857 \mathrm{MHz}$ \\
\hline
\end{tabular}

current mirror based CA has obtained good results for input current range and output linearity as to Wilson current mirror based CA. But, for DC analysis, the results of cascode mirror based CA have obtained approximately same values for input current range and output linearity. Finally, all of CM based CAs have got wide input current range as $\pm 200 \mu \mathrm{A}$. But, cascode CM based CAs are more linearity input response than Wilson CM based CAs.

The figure of overall frequency response obtained from the well-known current mirrors based CAs mentioned above is shown in Fig. 2. The Wilson current mirror based CA structures have got high frequency range to cascode current mirror based CA structures as shown in Fig. 2. Also, the Wilson current mirror based CA has obtained good result for cut-off frequency and frequency response. Finally, the specifications of the all CAs are summarized in Table I.

\section{Conclusion}

In this paper, the important characteristics of well-known (Wilson, improved 
Wilson, cascode, and modified cascode) MOS current mirrors based Current Amplifier (CA) configurations have been discussed. The proposed Current Amplifier (CA) topologies are presented and compared with some basic parameters such as power dissipation, bias current, input resistance, output resistance, and cut-off frequency by using PSPICE simulation experiments for HP $0.8 \mu \mathrm{m}$ CMOS process parameters. Finally, we intended to provide more information on the performance characteristics of current mirror based CA circuit blocks for integrated circuit designers. The main purpose of this study will present valuable brief information of details for analog circuit designers, and will help them in selecting the appropriate architecture about a current mirror based CA circuits.

The results obtained in this paper will be of primary importance to the designer selecting the $\mathrm{CA}$ to meet the requirements of the different performance aspects, especially output resistance. The essential findings of this paper are summarized in Table I, where identical MOS transistors and a unity current ratio are assumed.

\section{Acknowledgments}

This paper was partially supported by research project in Erciyes University, Turkey (Project No. FBT-04-28). 